\begin{abstract}
The paper stresses the relevance of polynomial matrices in three different approaches to the analysis of two-dimensional systems: input/output maps, state models and behavior descriptions. Some aspects of i/o and state models, like stability definitions and their algebraic characterizations, as well as conditions for the existence of stabilizing/dead-beat controllers are surveyed. Finally, some preliminary results about stability and the design of stabilizing controllers in the context of $2 \mathrm{D}$ behavior theory are presented.

Two-dimensional systems, input/output models, state-space models, behaviour, stability, stabilizing controllers
\end{abstract}




\title{
2D SYSTEMS ANALYSIS: A POLYNOMIAL APPROACH
}

\author{
E. Fornasini* and M.E. Valcher*
}

\section{INTRODUCTION}

The foundations of the polynomial matrix approach to linear systems, along with its applications to industrial control design, were laid down in the early seventies by H.H.Rosenbrock [?]. Since that time the theory has been developed by a large number of researchers, and nowadays polynomial matrix methods have gained a large acceptance within the control community. Among the others, one of the main advantages of this conceptual framework is the possibility of analysing and designing two-dimensional (2D) systems, both in their classical structure of quarter plane causal filters and in the modern behavioral setting, introduced by J.C.Willems. In fact, (Laurent) polynomial and rational matrices in two variables often constitute the only tool available for formulating and solving 2D problems, as state space methods, based on the geometric theory of finite dimensional spaces, do not extend to multidimensional systems.

The aim of this paper is to give some flavour of the kind of questions which have already been answered, and to indicate some directions for future investigations. We shall assume the reader to be acquainted with the material on 2D polynomial matrices presented in [?] and to have an elementary knowledge of basic 2D system theory, as presented, for instance, in [?].

Before proceeding, we introduce some notation. Throughout the paper we will denote by $\mathbb{R}\left[z_{1}, z_{2}\right], \mathbb{R}\left[z_{1}, z_{2}, z_{1}^{-1}, z_{2}^{-1}\right]$ and $\mathbb{R}\left(z_{1}, z_{2}\right)$ the rings of polynomials and Laurent polynomials (L-polynomials) and the field of rational functions, respectively, in the indeterminates $z_{1}$ and $z_{2}$, with coefficients in $\mathbb{R}$. For any two-dimensional sequence $\mathbf{w}=\{\mathbf{w}(h, k)\}_{h, k \in \mathbb{Z}} \in$ $\left(\mathbb{R}^{q}\right)^{\mathbb{Z} \times \mathbb{Z}}$, the support of $\mathbf{w}$ is the set of points $\operatorname{supp}(\mathbf{w}):=\{(h, k) \in$ $\mathbb{Z} \times \mathbb{Z}: \mathbf{w}(h, k) \neq 0\}$. As it is customary, any two-dimensional sequence $\mathbf{w}$ will be identified with the corresponding formal power series $\sum_{h, k \in \mathbb{Z}} \mathbf{w}(h, k) z_{1}^{h} z_{2}^{k}$, thus exploiting the bijective correspondence between sequences taking values in $\mathbb{R}^{q}$ and formal power series with coefficients in $\mathbb{R}^{q}$. For sake of brevity, the sequence space $\left(\mathbb{R}^{q}\right)^{\mathbb{Z} \times \mathbb{Z}}$ will be denoted by $\mathcal{R}_{\infty}^{q}$.

${ }^{*}$ Dip. di Elettronica ed Informatica, Univ. di Padova, Padova, ITALY 


\section{2D SYSTEMS MODELLING}

The simplest class of $2 \mathrm{D}$ systems are those in input/output (i/o) form, which represent the natural generalization of $1 \mathrm{D}$ input/output linear systems. Partitioning the system variables into "inputs" and "outputs", u and y respectively, aims to distinguish between "causes" and "effects" within the phaenomenon to describe. Usually, inputs are assumed $m$ dimensional and completely free, while outputs are $p$-dimensional and uniquely determined by the inputs by means of an input/output map.

Under the linearity, shift-invariance and finite-dimensionality assumptions, i/o maps are expressed by means of a $p \times m$ rational transfer matrix $W\left(z_{1}, z_{2}\right)=\sum_{(i, j) \in S} W_{i, j} z_{1}^{i} z_{2}^{j}$ as follows

$$
Y\left(z_{1}, z_{2}\right)=W\left(z_{1}, z_{2}\right) U\left(z_{1}, z_{2}\right)
$$

where $U\left(z_{1}, z_{2}\right)$ and $Y\left(z_{1}, z_{2}\right)$ are the formal power series corresponding to the input and output sequences $\mathbf{u}$ and $\mathbf{y}$. The set $S$ is a suitable, generally infinite, subset of $\mathbb{Z} \times \mathbb{Z}$. A typical assumption on this class of models is "quarter-plane causality", which amounts to saying that $S$ is included in the positive orthant $\{(h, k) \in \mathbb{Z} \times \mathbb{Z}: h \geq 0, k \geq 0\}$ or, equivalently, that in every left coprime matrix fraction description, $D^{-1}\left(z_{1}, z_{2}\right) N\left(z_{1}, z_{2}\right)$, of $W\left(z_{1}, z_{2}\right)$ we have $\operatorname{det} D(0,0) \neq 0$. Rational matrices endowed with this property are called proper.

An alternative approach to 2D systems is in terms of state-space description. By a 2D state-model we mean a quarter plane causal 2D system described by the following equations [?]:

$$
\begin{aligned}
\mathbf{x}(h+1, k+1) & =A_{1} \mathbf{x}(h, k+1)+A_{2} \mathbf{x}(h+1, k) \\
& +B_{1} \mathbf{u}(h, k+1)+B_{2} \mathbf{u}(h+1, k) \\
\mathbf{y}(h, k) & =C \mathbf{x}(h, k)+D \mathbf{u}(h, k),
\end{aligned}
$$

where $\mathbf{u}(\cdot, \cdot), \mathbf{x}(\cdot, \cdot)$ and $\mathbf{y}(\cdot, \cdot)$ are the input, state and output sequences, taking values in $\mathbb{R}^{m}, \mathbb{R}^{n}$ and $\mathbb{R}^{p}$ respectively, while $A_{1}, A_{2}, B_{1}, B_{2}, C$ and $D$ are real matrices of suitable dimensions. Initial conditions are assigned by specifying the state values $\mathbf{x}(h, k)$ on the separation set $\mathcal{S}_{0}:=\{(h, k) \in$ $\mathbb{Z} \times \mathbb{Z}: h+k=0\}$. So, when an input sequence $\mathbf{u}(\cdot, \cdot)$ has been assigned over the half-plane $\mathcal{H}_{0}^{+}:=\{(h, k) \in \mathbb{Z} \times \mathbb{Z}: h+k \geq 0\}$, the state and output sequences can be computed at every point of $\mathcal{H}_{0}^{+}$.

Upon representing input, state and output sequences by means of formal power series, model (2) is equivalently described as follows

$$
\begin{aligned}
& X\left(z_{1}, z_{2}\right)=\left(I-A_{1} z_{1}-A_{2} z_{2}\right)^{-1} \sum_{i} \mathbf{x}(i,-i) z_{1}^{i} z_{2}^{-i} \\
& \quad+\left(I-A_{1} z_{1}-A_{2} z_{2}\right)^{-1}\left(B_{1} z_{1}+B_{2} z_{2}\right) U\left(z_{1}, z_{2}\right) \\
& Y\left(z_{1}, z_{2}\right)=C X\left(z_{1}, z_{2}\right)+D U\left(z_{1}, z_{2}\right)
\end{aligned}
$$

and, if we assume zero initial conditions on $\mathcal{S}_{0}$, the associated i/o map is given by $Y\left(z_{1}, z_{2}\right)=W\left(z_{1}, z_{2}\right) U\left(z_{1}, z_{2}\right)$, where $W\left(z_{1}, z_{2}\right):=C\left(I-A_{1} z_{1}-\right.$ $\left.A_{2} z_{2}\right)^{-1}\left(B_{1} z_{1}+B_{2} z_{2}\right)+D$ represents the (quarter plane causal) transfer matrix of the $2 \mathrm{D}$ state model. 
Clearly, every 2D state model determines a unique transfer matrix, and hence a unique i/o map, while the inverse problem of "realizing" a given $2 \mathrm{D}$ proper rational matrix by means of a state model admits infinitely many solutions [?].

Quite recently, the behavior approach to the description of dynamical systems, introduced by J.C.Willems [?] has been extended to the multidimensional case [?, ?]. One of its main features is that it focuses its interest on the set of system trajectories, the behavior, while making no distinction between inputs and outputs when describing how the system interacts with its environment. Input/output and state space descriptions have to be deduced only later, from the mathematical equations, provided that the analysis of the behavior has enlightened some cause/effect structure.

In the $2 \mathrm{D}$ context a dynamical system $\Sigma$ is defined as a triple $\Sigma=$ $\left(\mathbb{Z} \times \mathbb{Z}, \mathbb{R}^{q}, \mathcal{B}\right)$, with $\mathbb{Z} \times \mathbb{Z}$ as independent variables set, $\mathbb{R}^{q}$ as the set where the system trajectories take values (the signal alphabet) and $\mathcal{B} \subseteq \mathcal{R}_{\infty}^{q}$ as the set of admissible trajectories (the behavior). In order to make it possible a comparison with the previous models, we must introduce the linearity and shift-invariance hypotheses also in the behavior descriptions. A further requirement on $\mathcal{B}$ is completeness [?, ?], which is the possibility of checking whether a sequence $\mathbf{w}$ belongs to the behavior by simply analysing its restrictions to the finite subsets of $\mathbb{Z} \times \mathbb{Z}$. In mathematical terms, the completeness of a behavior $\mathcal{B}$ corresponds to the existence of an L-polynomial matrix $H^{T}$ such that

$$
\mathcal{B}=\operatorname{ker} H^{T}:=\left\{\mathbf{w} \in \mathcal{R}_{\infty}^{q}: H^{T} \mathbf{w}=0\right\} .
$$

The most significant property a behavior can be endowed with is undoubtely zero-controllability [?, ?] which expresses the possibility of "embedding" any portion of a behavior trajectory into a new trajectory whose support slightly exceeds that of the available portion. More precisely, we say that a behavior $\mathcal{B}$ is zero-controllable if there exists a positive integer $\delta$ such that, for every finite set $\mathcal{T} \subset \mathbb{Z} \times \mathbb{Z}$ and every $\mathbf{w} \in \mathcal{B}$, there is a trajectory $\tilde{\mathbf{w}} \in \mathcal{B}$, which coincides with $\mathbf{w}$ in $\mathcal{T}$ and has support included in $\mathcal{T}^{\delta}:=\{(i, j) \in \mathbb{Z} \times \mathbb{Z}: d((i, j), \mathcal{T})<\delta\}$, where the distance $d((i, j), \mathcal{T})$ is defined as $\min \{|i-h|+|j-k| ;(h, k) \in \mathcal{T}\}$. Controllability induces a very peculiar polynomial matrix description for a behavior $\mathcal{B}$. $2 \mathrm{D}$ controllable behaviors, indeed, are kernels of left factor prime L-polynomial matrices or, equivalently, image spaces of suitable L-polynomial operators, i.e.

$$
\mathcal{B}=\operatorname{Im} G:=\left\{\mathbf{w} \in \mathcal{R}_{\infty}^{q}: \mathbf{w}=G \mathbf{u}, \mathbf{u} \in \mathcal{R}_{\infty}^{m}\right\},
$$

for some $G \in \mathbb{R}\left[z_{1}, z_{2}, z_{1}^{-1}, z_{2}^{-1}\right]^{q \times m}$.

A property which is somehow opposite to controllability is autonomy. A behavior $\mathcal{B}$ is autonomous if there exists a solid cone $\mathcal{C}$ in $\mathbb{R} \times \mathbb{R}$ such that the restriction of any behavior trajectory $\mathbf{w}$ to $\mathcal{C} \cap(\mathbb{Z} \times \mathbb{Z})$ allows to uniquely retrieve the remaining portion of $\mathbf{w}$. Autonomous behaviors are kernels of full column rank L-polynomial matrices [?].

Controllable and autonomous behaviors constitute the building blocks for constructing other behaviors, since every complete behavior $\mathcal{B}$ can be expressed [?] as the sum $\mathcal{B}=\mathcal{B}_{c}+\mathcal{B}_{a}$ of its "controllable part" $\mathcal{B}_{c}$, which is the maximal controllable behavior included in $\mathcal{B}$, and of a suitable autonomous behavior $\mathcal{B}_{a}$. 


\section{I/O AND STATE MODELS: STRUC- TURAL PROPERTIES AND CONTROL PROBLEMS}

When dealing with quarter-plane causal 2D i/o models, a fundamental issue is undoubtely represented by BIBO (bounded input/bounded output) stability. An i/o model is BIBO stable if it produces bounded output sequences when stimulated by bounded input sequences with support in $\mathcal{H}_{0}^{+}$or, equivalently, if the coefficients $W_{i, j}$ of its transfer matrix expansion $W\left(z_{1}, z_{2}\right)=\sum_{i, j \in \mathbb{N}} W_{i, j} z_{1}^{i} z_{2}^{j}$ constitute an $\ell_{1}$-sequence.

It has been shown [?] that a sufficient condition for BIBO stability is that $W\left(z_{1}, z_{2}\right)$ is devoid of singularities within the closed unit polydisk

$$
\overline{\mathcal{P}}_{1}:=\left\{\left(z_{1}, z_{2}\right) \in \mathbb{C} \times \mathbb{C}:\left|z_{1}\right| \leq 1,\left|z_{2}\right| \leq 1\right\} .
$$

On the other hand, when $W\left(z_{1}, z_{2}\right)$ has singularities in $\overline{\mathcal{P}}_{1} \backslash \mathcal{T}_{1}$, where

$$
\mathcal{T}_{1}:=\left\{\left(z_{1}, z_{2}\right) \in \mathbb{C} \times \mathbb{C}:\left|z_{1}\right|=1,\left|z_{2}\right|=1\right\}
$$

is the distinguished boundary of $\overline{\mathcal{P}}_{1}$, the i/o map is not BIBO stable. When $W\left(z_{1}, z_{2}\right)$ is regular in $\overline{\mathcal{P}}_{1} \backslash \mathcal{T}_{1}$ but exhibits (nonessential) singularities of the second kind in $\mathcal{T}_{1}$, a general result about BIBO stability is not available, as there are both examples of stable [?] and of unstable maps with these features. In any case, as transfer matrices with second kind singularities on $\mathcal{T}_{1}$ cannot be realized via internally stable state models, it is often convenient to strengthen the stability definition and consider BIBO stable only rational matrices devoid of singularities in $\overline{\mathcal{P}}_{1}$.

Under this assumption, the feedback stabilization problem (see [?] for a complete survey on this topic) has been stated in the following terms: given a "plant", described by some strictly proper rational transfer matrix $W\left(z_{1}, z_{2}\right) \in \mathbb{R}\left(z_{1}, z_{2}\right)^{p \times m}$, find a (proper) rational matrix $C\left(z_{1}, z_{2}\right) \in$ $\mathbb{R}\left(z_{1}, z_{2}\right)^{m \times p}$ such that the resulting connected system

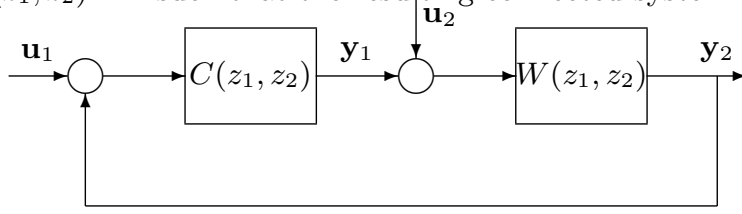

has an $(m+p) \times(m+p)$ proper stable rational transfer matrix.

If $D\left(z_{1}, z_{2}\right)^{-1} N\left(z_{1}, z_{2}\right)$ is a left coprime MFD of the plant transfer matrix $W\left(z_{1}, z_{2}\right)$, the plant is stabilizable by means of a proper rational compensator if and only if the variety of the maximal order minors of $\left[D\left(z_{1}, z_{2}\right) \mid N\left(z_{1}, z_{2}\right)\right]$ does not intersect $\overline{\mathcal{P}}_{1}$.

Structural properties of $2 \mathrm{D}$ state models constitute a much wider field of research. Actually, as stability is defined as a property of the state variables, feedback stabilization depends both on the way inputs affect the state of a system and on the possibility of reconstructing the state from the external variables. Therefore, a preliminary investigation of properties like local controllability, stabilizability, causal reconstructability and detectability is needed for dealing with the problem of designing dead-beat or stabilizing controllers. 
A 2D state model is internally stable if the free state evolution, corresponding to every set of bounded initial conditions on $\mathcal{S}_{0}$, asymptotically goes to zero, i.e. $\mathbf{x}(h, k) \rightarrow 0$ as $h+k \rightarrow+\infty$. As proved in [?], a $2 \mathrm{D}$ state model is internally stable if and only if the variety of its characteristic polynomial $\operatorname{det}\left(I-A_{1} z_{1}-A_{2} z_{2}\right)$ does not intersect $\overline{\mathcal{P}}_{1}$. It is clear that the internal stability of a state model ensures the BIBO stability of the associated i/o map, while the converse is not true, not even when dealing with minimal realizations.

A 2D state model is stabilizable if for any set of bounded initial conditions on $\mathcal{S}_{0}$, there exist real numbers $R>0$ and $\rho>1$ and an input sequence $\mathbf{u}(\cdot, \cdot)$, with support in $\mathcal{H}_{0}^{+}$, satisfying $\|\mathbf{u}(h, k)\|<\rho^{-(h+k)}$, $\forall h, k \in \mathcal{H}_{0}^{+}$, such that the corresponding state evolution satisfies

$$
\|\mathbf{x}(h, k)\|<R / \rho^{h+k}, \quad h, k \in \mathbb{N} .
$$

As shown in [?], stabilizability of 2D state models admits a polynomial matrix characterization which represents a natural extension of the wellknown PBH test for $1 \mathrm{D}$ systems. Actually, a 2D system is stabilizable if and only if

$$
\operatorname{rank}\left[I-A_{1} z_{1}-A_{2} z_{2} \mid B_{1} z_{1}+B_{2} z_{2}\right]=n,
$$

for every $\left(z_{1}, z_{2}\right) \in \overline{\mathcal{P}}_{1}$. Also detectability, i.e. the possibility of constructing an asymptotic observer for the $2 \mathrm{D}$ state model, can be characterized by means of a suitable $\mathrm{PBH}$ matrix. In fact, a $2 \mathrm{D}$ system is detectable if and only if [?]

$$
\operatorname{rank}\left[\frac{I-A_{1} z_{1}-A_{2} z_{2}}{C}\right]=n,
$$

for every $\left(z_{1}, z_{2}\right) \in \overline{\mathcal{P}}_{1}$.

The controller design problem in the context of $2 \mathrm{D}$ state-models is stated as an output feedback stabilization problem. More precisely, we look for a $2 \mathrm{D}$ state model, connected to the original plant as in the following picture

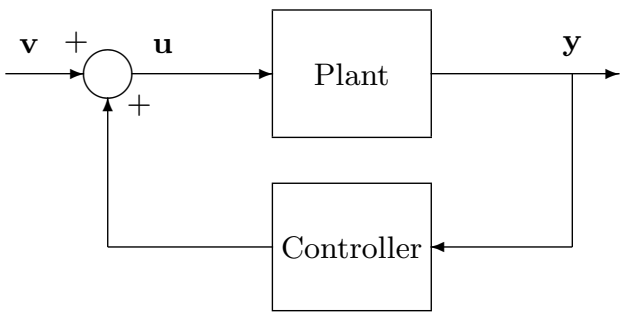

and making the overall system internally stable. A stabilizing controller exists if and only if the plant is both stabilizable and detectable, and, interestingly enough, these two conditions are the same ones guaranteeing the existence of a stabilizing regulator for a $1 \mathrm{D}$ system.

Local controllability and causal reconstructability appear as stronger versions of stabilizability and detectability [?, ?]. Indeed, a 2D state model is locally controllable if for any set of initial conditions on $\mathcal{S}_{0}$, there exist an input sequence $\mathbf{u}$, with support in $\mathcal{H}_{0}^{+}$, and a positive integer $N$, such that the state evolution satisfies $\mathbf{x}(h, k)=0$, for $h+k>N$, 
while causal reconstructability expresses the possibility of constructing a dead-beat observer.

Also these properties admit polynomial matrix characterizations, as they correspond to the cases when matrices (4) and (5) have full rank for every $\left(z_{1}, z_{2}\right) \in \mathbb{C} \times \mathbb{C}$ (i.e. are zero prime polynomial matrices). Moreover, local controllability and causal reconstructability are necessary and sufficient for the existence of dead-beat controllers.

\section{SOME RESULTS ON 2D BEHAVIORS STABILITY}

The relevance of L-polynomial matrices in investigating the structural properties of $2 \mathrm{D}$ behaviors was pointed out by several authors [?, ?]. As we have seen, important features, like completeness, autonomy and controllability, have a polynomial matrix characterization, and the same holds true for observability and extendability. Instead of presenting an overview of the available results, we prefer, however, to address further aspects of $2 \mathrm{D}$ behavior theory which are still unexplored. So, in this section we make a first attempt to introduce the notions of stability and stabilizability for $2 \mathrm{D}$ behaviors, and to relate them to the algebraic features of the L-polynomial matrices involved in their description.

Introducing the stability issue in the $2 \mathrm{D}$ context requires some preliminary assumptions. Indeed, stability of ordinary 1D systems naturally refers to the common interpretation of the independent variable $t$ as time coordinate, and consequently to the trajectory evolution as $t$ goes to $+\infty$. For $2 \mathrm{D}$ systems, instead, there is no natural ordering in the discrete grid $\mathbb{Z} \times \mathbb{Z}$, and hence no obvious "future direction" can be singled out in the system evolution. The quarter plane causality assumption for $2 \mathrm{D}$ i/o maps and state models leads to a stability notion that refers to the evolution of the output and state trajectories, respectively, on the separation sets $\mathcal{S}_{t}:=\{(h, k) \in \mathbb{Z} \times \mathbb{Z}: h+k=t\}$ as $t$ goes to $+\infty$.

The stability definition we are going to introduce is somehow tailored for analysing the dynamics of behavior trajectories on the separation sets $\mathcal{S}_{t}$ for increasing values of $t$. Although many alternative definitions could be given, this choice has the advantage of allowing for comparisons with the previous models, considered as special cases of $2 \mathrm{D}$ dynamical systems. Intuitively speaking, a behavior $\mathcal{B}$ should be called stable when, for every trajectory $\mathbf{w} \in \mathcal{B}$, we have $\mathbf{w}(h, k) \rightarrow 0$ as $h+k \rightarrow+\infty$. This rules out all behaviors with a nontrivial controllable part $\mathcal{B}_{c}$. In fact, as $\mathcal{B}_{c}$ includes a finite support trajectory $\mathbf{w}_{f} \neq 0$, there exists a pair of positive integers $(\ell, m)$ such that $\sum_{i \in \mathbb{N}} z_{1}^{i \ell} z_{2}^{i m} \mathbf{w}_{f}$ is an infinite support trajectory which does not estinguish asymptotically. Consequently, stability notion concerns only autonomous behaviors and among them, due to the assumptions on the system evolution, only those for which the cone $\mathcal{C}$, where the trajectories can be uniquely recognized, is included in $\mathcal{H}_{0}^{-}:=\{(h, k) \in \mathbb{Z} \times \mathbb{Z}: h+k<0\}$. This implies that we can always reconstruct a trajectory from its restriction to $\mathcal{H}_{0}^{-}$and this is possible, in particular, when the behavior $\mathcal{B}$ is the kernel of a polynomial matrix 
$H^{T} \in \mathbb{R}\left[z_{1}, z_{2}\right]^{m \times q}$ with $H^{T}(0,0)$ full column rank. For sake of simplicity, in this contribution we will afford only this special case.

Finally, in analogy with the definition of internal stability for state space models, it seems convenient to require that the asymptotic convergence of the local states is uniform w.r. to the separation sets. As a consequence, we will consider only behavior trajectories which are bounded on a suitable "strip" $\cup_{i=-M}^{-1} \mathcal{S}_{i}$.

Definition Let $\Sigma=\left(\mathbb{Z} \times \mathbb{Z}, \mathbb{R}^{q}, \mathcal{B}\right)$ be a system, endowed with a complete autonomous behavior $\mathcal{B}=\operatorname{ker} H^{T}, H^{T}$ an $m \times q$ polynomial matrix, with homogeneous degree $M$ and $H^{T}(0,0)$ of rank $q$. We say that $\mathcal{B}$ is stable if for every sequence $\mathbf{w} \in \mathcal{B}$, which is bounded on the "strip" $\cup_{i=-M}^{-1} \mathcal{S}_{i}$, we have $\mathbf{w}(h, k) \rightarrow 0$ as $h+k \rightarrow+\infty$.

As in the case of state space models, stability of an autonomous behavior $\mathcal{B}$ is related to the intersections of the closed unit polydisk $\overline{\mathcal{P}}_{1}$ with a suitable algebraic variety.

Proposition Let $\Sigma=\left(\mathbb{Z} \times \mathbb{Z}, \mathbb{R}^{q}, \mathcal{B}\right)$ be a system, endowed with a complete autonomous behavior $\mathcal{B}=\operatorname{ker} H^{T}, H^{T}$ an $m \times q$ polynomial matrix, with homogeneous degree $M$ and $H^{T}(0,0)$ of rank $q$. If the variety $\mathcal{V}\left(H^{T}\right)$ of the maximal order minors of $H^{T}$ does not intersect the closed unit polydisk $\overline{\mathcal{P}}_{1}$, then $\mathcal{B}$ is stable.

Proof As $\mathcal{V}\left(H^{T}\right) \cap \overline{\mathcal{P}}_{1}=\emptyset$, there exists also $\rho>1$ such that $\mathcal{V}\left(H^{T}\right) \cap$ $\overline{\mathcal{P}}_{\rho}=\emptyset$, with $\overline{\mathcal{P}}_{\rho}:=\left\{\left(z_{1}, z_{2}\right) \in \mathbb{C} \times \mathbb{C}:\left|z_{1}\right| \leq \rho,\left|z_{2}\right| \leq \rho\right\}$. Thus, the ideal generated by the maximal order minors $m_{i}\left(H^{T}\right)$ of $H^{T}$ includes a polynomial $p_{\rho}=\sum_{i} c_{i} m_{i}\left(H^{T}\right)$ whose variety $\mathcal{V}\left(p_{\rho}\right)$ does not intersect $\overline{\mathcal{P}}_{\rho}$. If $S_{i}$ denotes the selection matrix corresponding to $m_{i}\left(H^{T}\right)$, then $m_{i}\left(H^{T}\right) I=\operatorname{adj}\left(S_{i} H^{T}\right) S_{i} H^{T}$, and consequently

$$
L:=\sum_{i} c_{i} \operatorname{adj}\left(S_{i} H^{T}\right) S_{i}
$$

is a polynomial matrix satisfying $L H^{T}=p_{\rho} I$. Consider, now, a trajectory $\mathbf{w} \in \mathcal{B}$, which is bounded in $\cup_{i=-M}^{-1} \mathcal{S}_{i}$, and denote by $\mathbf{w}_{+}$and $\mathbf{w}_{-}$its restrictions to $\mathcal{H}_{0}^{+}$and $\mathcal{H}_{0}^{-}$, respectively. Then $H^{T}\left(\mathbf{w}_{+}+\mathbf{w}_{-}\right)=0$ implies that the sequence $\mathbf{s}:=H^{T} \mathbf{w}_{+}=-H^{T} \mathbf{w}_{-}$, and hence also $\tilde{\mathbf{s}}:=L \mathbf{s}$, are bounded sequences with support included in some strip. Let $\cup_{i=0}^{N-1} \mathcal{S}_{i}$, $N \geq M$, be a strip including $\operatorname{supp}(\tilde{\mathbf{s}})$ and $\operatorname{set} S:=\sup \{\|\tilde{\mathbf{s}}(i, j)\|, 0 \leq$ $i+j \leq N-1\}$. As $1 / p_{\rho}$ admits a power series expansion $\sum_{i, j \in \mathbb{N}} q_{i, j} z_{1}^{i} z_{2}^{j}$, which is absolutely convergent in $\overline{\mathcal{P}}_{\rho}$, there exists a positive real $K$ such that

$$
\sum_{t \leq i+j \leq t+N-1}\left|q_{i j}\right|<K / \rho^{t}, \quad \forall t \in \mathbb{N} .
$$

As a consequence, from $\tilde{\mathbf{s}}=L H^{T} \mathbf{w}_{+}=p_{\rho} \mathbf{w}_{+}$, it follows that the sequence $\mathbf{w}_{+}=\tilde{\mathbf{s}} / p_{\rho}$ satisfies

$$
\|\mathbf{w}(h, k)\| \leq \frac{S K \rho^{N-1}}{\rho^{h+k}}, \quad h+k \geq 0
$$

which proves stability.

Consider, now, a complete nonautonomous behavior $\mathcal{B}$ and a representation of $\mathcal{B}$ as the sum of its controllable part and an autonomous behavior 
$\mathcal{B}_{a}$, i.e. $\mathcal{B}=\mathcal{B}_{c}+\mathcal{B}_{a}$. Then, every trajectory $\mathbf{w}$ of $\mathcal{B}$ decomposes into a trajectory $\mathbf{w}_{c} \in \mathcal{B}_{c}$ and a trajectory $\mathbf{w}_{a} \in \mathcal{B}_{a}$. Replace now $\mathbf{w}_{c}$ with a trajectory $\mathbf{w}_{c}^{\prime} \in \mathcal{B}_{c}$ which coincides with $\mathbf{w}_{c}$ in $\mathcal{H}_{0}^{-}$and is zero for all $(h, k)$ with $h+k \geq \delta, \delta$ a suitable integer. If we assume, as before, that every trajectory in the autonomous part is uniquely determined by its restriction to the halfplane $\mathcal{H}_{0}^{-}$, it is immediate to realize that the possibility of asymptotically driving to zero a behavior trajectory only depends on the autonomous part $\mathcal{B}_{a}$. These arguments lead to the following definition of stabilizability.

Definition Let $\Sigma=\left(\mathbb{Z} \times \mathbb{Z}, \mathbb{R}^{q}, \mathcal{B}\right)$ be a system, endowed with a complete behavior $\mathcal{B}=\operatorname{ker} H^{T}, H^{T}$ an $m \times q$ polynomial matrix of rank $q$, with homogeneous degree $M$, and let $\mathcal{B}_{c}$ be the controllable part of $\mathcal{B}$. We say that $\mathcal{B}$ is stabilizable if there exists a decomposition $\mathcal{B}=\mathcal{B}_{c}+\mathcal{B}_{a}$, such that for every sequence $\mathbf{w} \in \mathcal{B}$, which can be expressed as $\mathbf{w}=\mathbf{w}_{c}+\mathbf{w}_{a}$, with $\mathbf{w}_{c} \in \mathcal{B}_{c}$ and $\mathbf{w}_{a} \in \mathcal{B}_{a}$ bounded on the "strip" $\cup_{i=-M}^{-1} \mathcal{S}_{i}$, there exists $\tilde{\mathbf{w}} \in \mathcal{B}$, which coincides with $\mathbf{w}$ on the halfplane $\mathcal{H}_{0}^{-}$and satisfies $\tilde{\mathbf{w}}(h, k) \rightarrow 0$ as $h+k \rightarrow+\infty$.

Proposition Let $\Sigma=\left(\mathbb{Z} \times \mathbb{Z}, \mathbb{R}^{q}, \mathcal{B}\right)$ be a system, endowed with a complete behavior $\mathcal{B}=\operatorname{ker} H^{T}, H^{T}$ an $m \times q$ (strictly) polynomial matrix of rank $r$, with homogeneous degree $M$, and assume that $H^{T}$ factors as $H^{T}=F \bar{H}^{T}$, with $F$ full column rank and $\bar{H}^{T}$ left factor prime polynomial matrices. If the variety $\mathcal{V}\left(H^{T}\right)$ and the variety of some maximal order minor of $\bar{H}^{T}, m_{i}\left(\bar{H}^{T}\right)$, do not intersect the closed unit polydisk $\overline{\mathcal{P}}_{1}$, then $\mathcal{B}$ is stabilizable.

Proof Let $\bar{H}_{1}^{T}$ be a maximal order submatrix of $\bar{H}^{T}$ such that $\mathcal{V}\left(\operatorname{det} \bar{H}_{1}^{T}\right) \cap \overline{\mathcal{P}}_{1}=\emptyset$, and assume, for instance, that it consists of the first columns of $\bar{H}^{T}$. Then, it is well-known [?] that $\mathcal{B}$ can be expressed as $\mathcal{B}=\mathcal{B}_{c}+\mathcal{B}_{a}$, with $\mathcal{B}_{c}=\operatorname{ker} \bar{H}^{T}$ and

$$
\mathcal{B}_{a}=\left\{\left[\begin{array}{c}
\mathbf{w}_{1} \\
0
\end{array}\right]: F \bar{H}_{1}^{T} \mathbf{w}_{1}=0\right\} .
$$

Clearly, as $\mathcal{B}_{a}$ is a stable autonomous behavior, $\mathcal{B}$ is stabilizable.

\section{OPEN PROBLEMS AND CONCLU- SIONS}

In this paper we have pointed out the relevance of the polynomial matrix approach in the study of $2 \mathrm{D}$ systems. To this end we have surveyed the three main models adopted for $2 \mathrm{D}$ systems description, and we have shown that, as far as i/o and state models are concerned, stability notion and other features, which arise in the stabilizing controller design problem, are completely captured by the algebraic properties of suitable polynomial matrices.

In the 2D behavioral setting several issues are still unexplored: in the previous section we have introduced the notions of stability and stabilizability, and we have made a first attempt to relate them to the polynomial matrices involved in the kernel descriptions of the behaviors. 
The next goal is that of designing a stabilizing controller. This problem can be afforded, as shown by J.C.Willems [?] in the 1D case, as an interconnection problem. More precisely, if $\Sigma=\left(\mathbb{Z} \times \mathbb{Z}, \mathbb{R}^{q}, \mathcal{B}\right)$ is a plant and $\Sigma_{c}=\left(\mathbb{Z} \times \mathbb{Z}, \mathbb{R}^{q}, \mathcal{B}_{c}\right)$ a controller, we define the controlled system as the system

$$
\Sigma \wedge \Sigma_{c}:=\left(\mathbb{Z} \times \mathbb{Z}, \mathbb{R}^{q}, \mathcal{B} \cap \mathcal{B}_{c}\right),
$$

obtained by interconnecting $\Sigma$ and $\Sigma_{c}$, namely the system whose trajectories obey to the laws of both systems simultaneously. The controlled system is described in the following picture, and it is important to notice that plant and controller play equivalent roles in the resulting connected system.

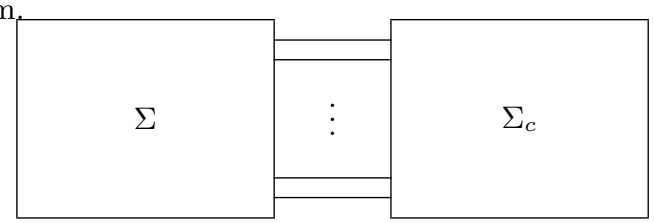

Although the above scheme may appear somehow restrictive, as, in general, plant and controller are connected only through certain terminals, all possible situations are easily reduced to the one just described, by resorting to a suitable redefinition of the system variables of plant and controller [?]. In this setting the stabilizing control problem is naturally stated in the following terms: given a plant $\Sigma=\left(\mathbb{Z} \times \mathbb{Z}, \mathbb{R}^{q}, \mathcal{B}\right)$ is it possible to design a controller $\Sigma_{c}=\left(\mathbb{Z} \times \mathbb{Z}, \mathbb{R}^{q}, \mathcal{B}_{c}\right)$ such that the resulting interconnected system is autonomous and stable?

For this and other problems connected with stability and stabilizability we refer the interested reader to a forthcoming paper [?].

\section{References}

[Anderson and Jury]Anderson and Jury1973Anderson-Jury Anderson, B.D.O. and E.I. Jury (1973). Stability test for two-dimensional recursive filters. IEEE Trans. Audio Electroacoustics AU-21, 366372. [Bisiacco]Bisiacco1985Mauro-stab Bisiacco, M. (1985). State and output feedback stabilizability of 2D systems. IEEE Trans. Circ. and Sys CAS 32, 1246-1249. [Bisiacco]Bisiacco1986Mauro-observers Bisiacco, M. (1986). On the structure of 2D observers. IEEE Trans. Aut. Contr. AC-31, 676-680. [Fornasini and Marchesini]Fornasini and Marchesini1976EF-GM-76 Fornasini, E. and G. Marchesini (1976). State space realization theory of two-dimensional filters. IEEE Trans. Aut. Contr. AC-21, 484 -92. [Fornasini and Marchesini]Fornasini and Marchesini1978doublyindexed Fornasini, E. and G. Marchesini (1978). Doubly indexed dynamical systems. Math.Sys. Theory 12, 59-72. [Fornasini and Valcher]Fornasini and Valcher1994IEEE Fornasini, E. and M.E. Valcher (1994). Algebraic aspects of 2D convolutional codes. IEEE Trans.Info.Th. IT 33, 12101225. [Fornasini and Valcher]Fornasini and Valcher1997behavior-stab Fornasini, E. and M.E. Valcher (1997). Stability and stabilizability of 2D behaviors. in preparation. [Fornasini et al.]Fornasini et al.1993EF- 
PR-SZ Fornasini, E., P. Rocha and S. Zampieri (1993). State realization of 2D finite dimensional autonomous systems. SIAM J. Contr. Optimiz. 31, 1502-1517. [Goodman] Goodman1977Goodman Goodman, D. (1977). Some stability properties of two-dimensional linear shift-invariant digital filters. IEEE Trans. Circ. Sys. CAS24, 201-208. [Guiver and Bose]Guiver and Bose1985Guiver-Bose Guiver, J.P. and N.K. Bose (1985). Causal and weakly causal 2-D filters with applications in stabilization. In: Multidimensional Systems Theory (N.K.Bose, Ed.). pp. 52-100. D.Reidel Publ. Co., Dordrecht (NL). [Kaczorek]Kaczorek1985Kaczorek85 Kaczorek, T. (1985). Two-dimensional linear systems. Lecture Notes in Control and INformation Sciences, Springer verlag, Berlin. [Morf et al.]Morf et al.1977Morf-Levy Morf, M., B.C. Lévy, S.Y. Kung and T. Kailath (1977). New results in 2D systems theory, part I and II. Proc. of IEEE 65, no.6, 861-872;945-961. [Rocha]Rocha1990Rocha Rocha, P. (1990). Structure and Representation of 2-D Systems. PhD thesis. University of Groningen, The Netherlands. [Rosenbrock]Rosenbrock1970Rosenbrock Rosenbrock, H.H. (1970). State-space and multivariable theory. J.Wiley \& Sons, New York. [Willems]Willems1988Willems Willems, J.C. (1988). Models for dynamics. Dynamics reported 2, 171-269. [Willems]Willems1996WillemsB Willems, J.C. (1996). On interconnections, control, and feedback. to appear in IEEE Trans. Aut. Contr. 